\title{
Bound and anti-bound soliton states for a quantum integrable derivative nonlinear Schrödinger model
}

\author{
B. Basu-Mallick ${ }^{1 *}$, Tanaya Bhattacharyya ${ }^{1 \dagger}$ and Diptiman Sen ${ }^{2 \ddagger}$ \\ ${ }^{1}$ Theory Group, \\ Saha Institute of Nuclear Physics, \\ 1/AF Bidhan Nagar, Kolkata 700 064, India \\ ${ }^{2}$ Centre for Theoretical Studies, Indian Institute of Science, \\ Bangalore 560012, India
}

\begin{abstract}
We find that localized quantum $N$-body soliton states exist for a derivative nonlinear Schrödinger (DNLS) model within an extended range of coupling constant $\left(\xi_{q}\right)$ given by $0<\left|\xi_{q}\right|<\frac{1}{\hbar} \tan \left(\frac{\pi}{N-1}\right)$. We also observe that soliton states with both positive and negative momentum can appear for a fixed value of $\xi_{q}$. Thus the chirality property of classical DNLS solitons is not preserved at the quantum level. Furthermore, it is found that the solitons with positive (negative) chirality have positive (negative) binding energy.
\end{abstract}

\footnotetext{
*e-mail address: biru@theory.saha.ernet.in

$\dagger$ e-mail address: tanaya@theory.saha.ernet.in

$\ddagger$ e-mail address: diptiman@cts.iisc.ernet.in
} 


\section{Introduction}

Quantum soliton states associated with integrable field models in $1+1$ dimension have attracted a lot of interest in recent years [1-10]. These quantum soliton states are usually constructed by using the approach of either the coordinate Bethe ansatz or the algebraic Bethe ansatz. By applying the coordinate Bethe ansatz to an integrable nonrelativistic Hamiltonian, one can directly obtain its exact eigenfunctions in the coordinate representation. If such an eigenfunction decays sufficiently fast when a relative coordinate measuring interparticle distance tends towards infinity, we define this localized square-integrable eigenfunction as a quantum soliton state. It is also possible to construct quantum soliton states through the approach of the algebraic Bethe ansatz, by choosing appropriate distributions of the spectral parameter on the complex plane [2-4]. The stability of quantum soliton states, in the presence of small external perturbations, can be measured by calculating their binding energy. It is usually found that localized quantum soliton states of various integrable models, including the well known nonlinear Schrödinger model and sine-Gordon model, have positive binding energy [1-5].

The purpose of the present article is to study the quantum soliton states of an integrable derivative nonlinear Schrödinger (DNLS) model [6-10]. Classical and quantum versions of DNLS model have found applications in different areas of physics like circularly polarized nonlinear Alfven waves in a plasma [11,12], quantum properties of the optical solitons in fibers [13] and some chiral Luttinger liquids which are obtained by dimensional reduction of a Chern-Simons model defined in two dimensions [14-15]. It is well known that the classical DNLS model can have solitons with momenta in only one direction [16-18]. Here we want to investigate whether this chirality property of the classical solitons is preserved at the quantum level. The Hamiltonian of quantum DNLS model in its second quantized form is given by $[6,7]$

$$
H=\hbar \int_{-\infty}^{+\infty} d x\left[\psi_{x}^{\dagger}(x) \psi_{x}(x)+i \xi_{q}\left\{\psi^{\dagger^{2}}(x) \psi(x) \psi_{x}(x)-\psi_{x}^{\dagger}(x) \psi^{\dagger}(x) \psi^{2}(x)\right\}\right]
$$

where we have set the particle mass $m=1 / 2, \xi_{q}$ is the coupling constant, and $\psi(x), \psi^{\dagger}(x)$ are field operators obeying the following equal time commutation relations: $[\psi(x), \psi(y)]=\left[\psi^{\dagger}(x), \psi^{\dagger}(y)\right]=0,\left[\psi(x), \psi^{\dagger}(y)\right]=\hbar \delta(x-y)$. Quantum soliton states of this DNLS model have been constructed through the approach of the alge-

braic Bethe ansatz [8-10] as well as the coordinate Bethe ansatz [6,7]. By applying the coordinate Bethe ansatz, it is found that quantum $N$-body soliton (henceforth called $N$-soliton) states exist for this DNLS model provided that $\xi_{q}$ is restricted within the 
range: $0<\left|\xi_{q}\right|<\frac{1}{\hbar} \tan \left(\frac{\pi}{N}\right)$. Moreover it is observed that, similar to the classical case, such $N$-soliton states can have only positive momentum for the case $\xi_{q}>0$ [6]. But, it was mentioned later that, soliton states can exist even for negative momentum for small values of $N$ [7]. However, no explicit range of $\xi_{q}$ is known for which such $N$-solitons with negative momenta may appear in the quantum DNLS model.

In this article we reinvestigate the range of $\xi_{q}$ for which localized quantum $N$ soliton states exist in the case of DNLS model. In Sec. 2, we apply the coordinate Bethe ansatz for this purpose and show that quantum $N$-soliton states exist within an extended range given by $0<\left|\xi_{q}\right|<\frac{1}{\hbar} \tan \left(\frac{\pi}{N-1}\right)$. We also find that the momentum of the $N$-soliton state has the same sign as the coupling constant when it lies within the range $0<\left|\xi_{q}\right|<\frac{1}{\hbar} \tan \left(\frac{\pi}{N}\right)$ and has the opposite sign to the coupling constant when $\frac{1}{\hbar} \tan \left(\frac{\pi}{N}\right)<\left|\xi_{q}\right|<\frac{1}{\hbar} \tan \left(\frac{\pi}{N-1}\right)$. As a result, soliton states with both positive and negative momentum can appear for a fixed value of $\xi_{q}$. In Sec. 3 we find out the binding energy of these soliton states. Sec. 4 is the concluding section.

\section{Quantum N-soliton states for DNLS model}

For applying the coordinate Bethe ansatz, we separate the full bosonic Fock space associated with Hamiltonian (1.1) into disjoint N-particle subspaces $\left|F_{N}\right\rangle$. We are interested in solving the eigenvalue equation $H\left|F_{N}\right\rangle=E\left|F_{N}\right\rangle$. The coordinate representation of this equation yields

$$
H_{N} \tau_{N}\left(x_{1}, x_{2}, \cdots, x_{N}\right)=E \tau_{N}\left(x_{1}, x_{2}, \cdots, x_{N}\right)
$$

where the N-particle symmetric wave function $\tau_{N}\left(x_{1}, x_{2}, \cdots, x_{N}\right)$ is defined as

$$
\tau_{N}\left(x_{1}, x_{2}, \cdots, x_{N}\right)=1 / \sqrt{n !}\left\langle 0\left|\psi\left(x_{1}\right) \cdots \psi\left(x_{N}\right)\right| F_{N}\right\rangle
$$

and $H_{N}$, the projection of the second-quantized Hamiltonian $H(1.1)$ on the N particle sector, is given by

$$
H_{N}=-\hbar^{2} \sum_{j=1}^{N} \frac{\partial^{2}}{\partial x_{j}^{2}}+2 i \hbar^{3} \xi_{q} \sum_{l<m} \delta\left(x_{l}-x_{m}\right)\left(\frac{\partial}{\partial x_{l}}+\frac{\partial}{\partial x_{m}}\right) .
$$

It is evident that this $H_{N}$ commutes with the total momentum operator in the $N$ particle sector, which is defined as

$$
P_{N}=-i \hbar \sum_{j=1}^{N} \frac{\partial}{\partial x_{j}}
$$


Next, we divide the coordinate space $R^{N} \equiv\left\{x_{1}, x_{2}, \cdots x_{N}\right\}$ into various $N$-dimensional sectors defined through inequalities like $x_{\omega(1)}<x_{\omega(2)}<\cdots<x_{\omega(N)}$, where $\omega(1), \omega(2)$, $\cdots, \omega(N)$ represents a permutation of $1,2, \cdots, N$. Within each such sector the interaction potential of the Hamiltonian (2.3) is zero and the resulting eigenfunction is just a superposition of the free particle wave functions. Coefficients associated with these free particle wave functions can be obtained through the interaction part of the Hamiltonian (2.3), which is nontrivial at the boundary of two adjacent sectors. It is known that all such necessary coefficients for the Bethe ansatz solution of a $N$-particle system can be obtained by solving the corresponding two particle problem [19]. Hence let us first try to construct the eigenfunctions of the Hamiltonian (2.3) for the two particle case, without imposing any symmetry property on $\tau_{2}\left(x_{1}, x_{2}\right)$ under the exchange of particle coordinates. For $x_{1}<x_{2}$ region, we may take such eigenfunction as

$$
\tau_{2}\left(x_{1}, x_{2}\right)=\exp \left\{i\left(k_{1} x_{1}+k_{2} x_{2}\right)\right\}
$$

where $k_{1}$ and $k_{2}$ are two distinct wave numbers. Using eqn. (2.1) for $N=2$, one can show that this two-particle wave function will take the following form in the region $x_{1}>x_{2}$ :

$$
\tau_{2}\left(x_{1}, x_{2}\right)=A\left(k_{1}, k_{2}\right) \exp \left\{i\left(k_{1} x_{1}+k_{2} x_{2}\right)\right\}+B\left(k_{1}, k_{2}\right) \exp \left\{i\left(k_{2} x_{1}+k_{1} x_{2}\right)\right\}
$$

where the 'matching coefficients' $A\left(k_{1}, k_{2}\right)$ and $B\left(k_{1}, k_{2}\right)$ are given by

$$
A\left(k_{1}, k_{2}\right)=\frac{\left(k_{1}-k_{2}\right)+i \hbar \xi_{q}\left(k_{1}+k_{2}\right)}{\left(k_{1}-k_{2}\right)}, \quad B\left(k_{1}, k_{2}\right)=1-A\left(k_{1}, k_{2}\right) .
$$

By using these matching coefficients, one can construct completely symmetric Nparticle eigenfunctions for the Hamiltonian (2.3). In the region $x_{1}<x_{2}<\cdots<x_{N}$, such eigenfunctions are given by $[6,19]$

$$
\tau_{N}\left(x_{1}, x_{2}, \cdots, x_{N}\right)=\sum_{\omega}\left(\prod_{l<m} \frac{A\left(k_{\omega(m)}, k_{\omega(l)}\right)}{A\left(k_{m}, k_{l}\right)}\right) \rho_{\omega(1), \omega(2), \cdots, \omega(N)}\left(x_{1}, x_{2}, \cdots, x_{N}\right),
$$

where

$$
\rho_{\omega(1), \omega(2), \cdots, \omega(N)}\left(x_{1}, x_{2}, \cdots, x_{N}\right)=\exp \left\{i\left(k_{\omega(1)} x_{1}+\cdots+k_{\omega(N)} x_{N}\right)\right\}
$$

In the expression $(2.8), k_{n}$ s are all distinct wave numbers and $\sum_{\omega}$ implies summing over all permutations of the numbers $(1,2, \ldots . \mathrm{N})$. The eigenvalues of the momentum $(2.4)$ and Hamiltonian (2.3) operators, corresponding to the eigenfunctions $\tau_{N}\left(x_{1}, x_{2}, \cdots, x_{N}\right)$, 
are given by

$$
\begin{aligned}
& P_{N} \tau_{N}\left(x_{1}, x_{2}, \cdots, x_{N}\right)=\hbar\left(\sum_{j=1}^{N} k_{j}\right) \tau_{N}\left(x_{1}, x_{2}, \cdots, x_{N}\right), \\
& H_{N} \tau_{N}\left(x_{1}, x_{2}, \cdots, x_{N}\right)=\hbar^{2}\left(\sum_{j=1}^{N} k_{j}^{2}\right) \tau_{N}\left(x_{1}, x_{2}, \cdots, x_{N}\right) .
\end{aligned}
$$

The wave function (2.8) will represent a localized soliton state, if it decays when any of the relative coordinates measuring an interparticle distance tends towards infinity. To obtain the condition for the existence of such localized soliton state, let us consider the following wave function in the region $x_{1}<x_{2}<\cdots<x_{N}$ :

$$
\rho_{1,2, \cdots, N}\left(x_{1}, x_{2}, \cdots, x_{N}\right)=\exp \left(i \sum_{j=1}^{N} k_{j} x_{j}\right) .
$$

As before, the momentum eigenvalue corresponding to this wave function is given by $\hbar \sum_{j=1}^{N} k_{j}$. Since this momentum eigenvalue must be a real quantity, one gets the condition

$$
\sum_{j=1}^{N} q_{j}=0
$$

where $q_{j}$ denotes the imaginary part of $k_{j}$. The probability density corresponding to the wave function $\rho_{1,2, \cdots, N}\left(x_{1}, x_{2}, \cdots, x_{N}\right)(2.11)$ can be expressed as

$$
\left|\rho_{1,2, \cdots, N}\left(x_{1}, x_{2}, \cdots, x_{N}\right)\right|^{2}=\exp \left\{2 \sum_{r=1}^{N-1}\left(\sum_{j=1}^{r} q_{j}\right) y_{r}\right\},
$$

where the $y_{r}$ 's are the $N-1$ number of relative coordinates: $y_{r} \equiv x_{r+1}-x_{r}$. It is evident that this probability density (2.13) decays exponentially in the limit $y_{r} \rightarrow \infty$ provided that all the following conditions are satisfied:

$$
q_{1}<0, \quad q_{1}+q_{2}<0, \quad \cdots \cdots, \sum_{j=1}^{N-1} q_{j}<0 .
$$

Note that the wave function (2.11) is obtained by taking $\omega$ as the identity permutation in (2.9). However, the full wave function (2.8) also contains terms like (2.9) with $\omega$ representing all possible nontrivial permutations. The conditions which ensure the decay of such a term with nontrivial permutation, in general, contradict the conditions (2.14). Consequently, to have an overall decaying wave function (2.8), the coefficients of all terms $\rho_{\omega(1), \omega(2), \cdots, \omega(N)}\left(x_{1}, x_{2}, \cdots, x_{N}\right)$ with nontrivial permutations are required to vanish. This requirement leads to a set of relations like

$$
A\left(k_{1}, k_{2}\right)=0, \quad A\left(k_{2}, k_{3}\right)=0, \cdots \cdots, A\left(k_{N-1}, k_{N}\right)=0 .
$$


Thus the simultaneous validity of the conditions (2.12), (2.14) and (2.15) ensures that the full wave function $\tau_{N}\left(x_{1}, x_{2}, \cdots, x_{N}\right)(2.8)$ would represent a localized soliton state. Using the conditions (2.12) and (2.15), one can derive the expression for the complex $k_{n}$ 's as

$$
k_{n}=\chi e^{-i(N+1-2 n) \phi}
$$

where $\chi$ is an yet undetermined real parameter, and $\phi$ is another real parameter which is related to the coupling constant as

$$
\phi=\tan ^{-1}\left(\hbar \xi_{q}\right)
$$

To obtain a unique value of $\phi$ from the above equation, we restrict it within the fundamental region: $-\frac{\pi}{2}<\phi<\frac{\pi}{2}$. In this context, it may be mentioned that a relation equivalent to (2.16) can also be obtained through the method of the algebraic Bethe ansatz, when quantum soliton states of DNLS model are considered [8-10]. Next, let us verify whether these $k_{n} \mathrm{~s}$ (2.16) satisfy the conditions (2.14). Summing over the imaginary parts of these $k_{n} \mathrm{~s}$, we can express the conditions (2.14) in the form

$$
\chi \frac{\sin \phi l}{\sin \phi} \sin \{\phi(N-l)\}>0,
$$

where $l \in[1,2,3, \cdots, N-1]$. For the simplest case $N=2$, the above condition is satisfied when $\phi$ lies within the range $0<\phi<\frac{\pi}{2}\left(-\frac{\pi}{2}<\phi<0\right)$ for the choice $\chi>0$ $(\chi<0)$. Thus any value of $\phi$ within its fundamental region can generate a 2 -soliton state. Next, we consider the case where $N \geq 3$. It may be noted that, $\sin \phi, \sin \phi l$, and $\sin \{\phi(N-l)\}$ are all positive (negative) when $\phi$ takes value within the range $0<\phi<$ $\frac{\pi}{N-1}\left(-\frac{\pi}{N-1}<\phi<0\right)$. Hence the condition (2.18) will be satisfied for the choice $\chi>0$ $(\chi<0)$, when $\phi$ lies within the range $0<\phi<\frac{\pi}{N-1}\left(-\frac{\pi}{N-1}<\phi<0\right)$. Due to eqn. (2.17), the corresponding coupling constant $\xi_{q}$ lies within the range $0<\xi_{q}<\frac{1}{\hbar} \tan \frac{\pi}{N-1}$ $\left(-\frac{1}{\hbar} \tan \frac{\pi}{N-1}<\xi_{q}<0\right)$. As a result, we get localized $N$-soliton states within the above mentioned range of $\phi$ or $\xi_{q}$. Earlier it has been shown that $N$-soliton state can be obtained for the case $\xi_{q}>0$ when $\phi$ lies within the range $0<\phi<\frac{\pi}{N}[6]$. The present analysis yields a wider range of $\phi$ for which a $N$-soliton state can be constructed.

Next, by using eqns. (2.16) and (2.10a), we obtain the momentum eigenvalue $(P)$ corresponding to these soliton states as

$$
P=\hbar \chi \frac{\sin \phi N}{\sin \phi} .
$$

Thus, for any given value of $\phi$, the momentum of such $N$-soliton state is proportional to the value of parameter $\chi$. Due to eqn. (2.19), it is evident that $P$ and $\chi$ have the 
same sign when $\phi$ lies in the range $0<|\phi|<\frac{\pi}{N}$ and the opposite sign when $\phi$ lies in the range $\frac{\pi}{N}<|\phi|<\frac{\pi}{N-1}$. However, we have seen earlier that $\chi$ and $\xi_{q}$ must have the same sign within the allowed range of a $N$-soliton state. Consequently, the momentum of the $N$-soliton state has the same sign as $\xi_{q}$ when $0<|\phi|<\frac{\pi}{N}$ and has the opposite sign to $\xi_{q}$ when $\frac{\pi}{N}<|\phi|<\frac{\pi}{N-1}$. We may call solitons with momenta having the same (opposite) sign as $\xi_{q}$ as solitons with positive (negative) chirality. It may be noted that the range of $\phi$ given by $\frac{\pi}{N}<|\phi|<\frac{\pi}{N-1}$ does not lie within its fundamental region for the case $N=2$. As a result, $N$-soliton states with negative chirality can appear only for $N \geq 3$.

The appearance of both positive and negative momentum solitons in quantum DNLS model can be described most conveniently by considering a fixed positive value of $\phi$ or $\xi_{q}$. Let us first take a fixed value of $\phi$ so that $\frac{\pi}{\phi}$ is an integer. In this case we obtain solitons with positive momentum for the choice $N=2,3, \cdots, \frac{\pi}{\phi}-1$ and a single soliton with zero momentum for $N_{\max }=\frac{\pi}{\phi}$. Next we consider the case where $\frac{\pi}{\phi}$ is non-integer and denote its integral part by $\left[\frac{\pi}{\phi}\right]$. In this case we obtain solitons with positive momentum for the choice $N=2,3, \cdots,\left[\frac{\pi}{\phi}\right]$ and a single soliton with negative momentum for $N_{\max }=\left[\frac{\pi}{\phi}\right]+1$. Thus we find that the chirality property of classical DNLS solitons is broken at the quantum level. It may be further noted that the ratio of solitons with positive and negative momentum is of the order of $\frac{\pi}{\phi}$. Moreover, due to eqn. (2.17), we get $\phi \rightarrow 0$ at the classical $\hbar \rightarrow 0$ limit (for any fixed value of $\xi_{q}$ ). As a result, the ratio of solitons with positive and negative momentum becomes infinitely large at the classical limit.

\section{$3 \quad$ Binding energy of a $N$-soliton state}

Here we want to calculate the binding energy for the $N$-soliton states of quantum DNLS model within the allowed range of $\phi$ given by $0<|\phi|<\frac{\pi}{N-1}$. Substituting the values of complex $k_{n}(2.16)$ in $(2.10 \mathrm{~b})$, we obtain the energy eigenvalue $(E)$ corresponding to these $N$-soliton states as

$$
E=\frac{\hbar^{2} \chi^{2} \sin (2 \phi N)}{\sin (2 \phi)}
$$

To calculate binding energy, we assume that the momentum $P(2.19)$ of the $N$-soliton state is equally distributed among $N$ number of single-particle scattering states. The real wave number associated with each of these single-particle states is denoted by $k_{0}$. 
With the help of eqns. (2.10a) and (2.19), we obtain

$$
k_{0}=\frac{\chi \sin (\phi N)}{N \sin \phi}
$$

Using eqn. (2.10b), one can easily calculate the total energy for $N$ number of such single-particle scattering states as

$$
E^{\prime}=\hbar^{2} N k_{0}^{2}=\frac{\hbar^{2} \chi^{2} \sin ^{2} \phi N}{N \sin ^{2} \phi}
$$

Subtracting $E$ (3.1) from $E^{\prime}$ (3.3), we obtain the binding energy of quantum $N$-soliton state as

$$
E_{B}(\phi, N) \equiv E^{\prime}-E=\frac{\hbar^{2} \chi^{2} \sin \phi N}{\sin \phi}\left\{\frac{\sin \phi N}{N \sin \phi}-\frac{\cos \phi N}{\cos \phi}\right\} .
$$

It may be noted that the above expression of binding energy remains invariant under the transformation $\phi \rightarrow-\phi$. Thus it is sufficient to investigate the nature of this binding energy only for positive values of $\phi$ within the range $0<\phi<\frac{\pi}{N-1}$. Substituting $N=2$ to eqn. (3.4), we obtain $E_{B}(\phi, 2)=2 \hbar^{2} \chi^{2} \sin ^{2} \phi$. Thus we get $E_{B}(\phi, 2)>0$ for any nonzero value of $\phi$. For $\mathrm{N}=3$, eqn. (3.4) takes the form $E_{B}(\phi, 3)=\frac{8 \hbar^{2} \chi^{2}}{3} \sin ^{2} \phi(3-$ $\left.4 \sin ^{2} \phi\right)$. Here we get $E_{B}(\phi, 3)>0$ when $0<\phi<\frac{\pi}{3}$ and $E_{B}(\phi, 3)<0$ when $\frac{\pi}{3}<\phi<\frac{\pi}{2}$. So from the above examples we can guess that $E_{B}(\phi, N)>0$ when $0<\phi<\frac{\pi}{N}$ and $E_{B}<0$ when $\frac{\pi}{N}<\phi<\frac{\pi}{N-1}$. By using the method of induction, we will try to prove that this condition is really valid for arbitrary values of $N$. To this end, we write $E_{B}(\phi, N)(3.4)$ in the form

$$
E_{B}(\phi, N)=\frac{\hbar^{2} \chi^{2} \sin \phi N}{\sin \phi} f(\phi, N)
$$

where

$$
f(\phi, N)=\frac{\sin \phi N}{N \sin \phi}-\frac{\cos \phi N}{\cos \phi} .
$$

To use the method of induction, let us first assume that $f(\phi, N)>0$, i.e.

$$
\frac{\sin \phi N}{\sin \phi}>\frac{N \cos \phi N}{\cos \phi}
$$

when $\phi$ lies within the range $0<\phi<\frac{\pi}{N}$. Next we consider an identity given by

$$
\frac{\sin \phi(N+1)}{\sin \phi}=\left(\frac{\sin \phi N}{\sin \phi}\right) \cos \phi+\cos \phi N .
$$

Using the inequality (3.7) and the above identity, we obtain

$$
\frac{\sin \phi(N+1)}{\sin \phi}>(N+1) \cos \phi N,
$$


for $0<\phi<\frac{\pi}{N}$. Next we consider another identity given by

$$
\cos \phi N=\frac{\cos \phi(N+1)}{\cos \phi}+\frac{\sin \phi \sin (\phi N)}{\cos \phi}
$$

Since $\sin \phi \sin (\phi N) / \cos \phi>0$ for $0<\phi<\frac{\pi}{N}$, the above identity yields

$$
(N+1) \cos \phi N>\frac{(N+1) \cos \phi(N+1)}{\cos \phi},
$$

for $0<\phi<\frac{\pi}{N}$. Now, combining the inequalities (3.8) and (3.9), we get

$$
\frac{\sin \phi(N+1)}{\sin \phi}>\frac{(N+1) \cos \phi(N+1)}{\cos \phi},
$$

for $0<\phi<\frac{\pi}{N}$. Thus by assuming that $f(\phi, N)>0$ within the range $0<\phi<\frac{\pi}{N}$, we are able to show that $f(\phi, N+1)>0$ within the same range of $\phi$. By putting $N=2$ in eqn. (3.6), it is easy to check that $f(\phi, 2)>0$ for $0<\phi<\frac{\pi}{2}$. Thus by using the method of induction, it is established that $f(\phi, N)>0$ within the range $0<\phi<\frac{\pi}{N-1}$ for all $N \geq 3$. Now, it may be noted that $\frac{\sin \phi N}{\sin \phi}>0$ for $0<\phi<\frac{\pi}{N}$. Consequently, from eqn. (3.5) we find that the binding energy for a $N$-soliton state of quantum DNLS model is positive when $\phi$ lies in the range $0<\phi<\frac{\pi}{N}$. However, it may also be noted that $\frac{\sin \phi N}{\sin \phi}<0$ within the range $\frac{\pi}{N}<\phi<\frac{\pi}{N-1}$. As a result, from eqn. (3.5) we interestingly find that the binding energy for a $N$-soliton state is negative when $\phi$ lies in the range $\frac{\pi}{N}<\phi<\frac{\pi}{N-1}$. Thus we find examples of quantum solitons in an integrable model where the binding energy can be negative.

\section{Concluding Remarks}

By applying the coordinate Bethe ansatz, we have investigated the range of the coupling constant $\xi_{q}$ for which localized quantum $N$-soliton states exist for DNLS model. It turns out that such quantum $N$-soliton states exist within an extended range of $\xi_{q}$ given by $0<\left|\xi_{q}\right|<\frac{1}{\hbar} \tan \left(\frac{\pi}{N-1}\right)$. We also observe that the $N$-soliton state has positive chirality when $\xi_{q}$ lies within the range $0<\left|\xi_{q}\right|<\frac{1}{\hbar} \tan \left(\frac{\pi}{N}\right)$ and negative chirality when $\frac{1}{\hbar} \tan \left(\frac{\pi}{N}\right)<\left|\xi_{q}\right|<\frac{1}{\hbar} \tan \left(\frac{\pi}{N-1}\right)$. Thus we find that the chirality property of classical DNLS solitons is not preserved at the quantum level. We also calculate the binding energy for the quantum soliton states of this model. Interestingly, it is found that the binding energy for a $N$-soliton state is positive when $0<\left|\xi_{q}\right|<\frac{1}{\hbar} \tan \left(\frac{\pi}{N}\right)$ and negative when $\frac{1}{\hbar} \tan \left(\frac{\pi}{N}\right)<\left|\xi_{q}\right|<\frac{1}{\hbar} \tan \left(\frac{\pi}{N-1}\right)$. Thus we can conclude that the solitons with positive chirality form bound states with positive binding energy. On the 
other hand, solitons with negative chirality have negative binding energy and hence they form anti-bound states. Consequently, solitons with negative chirality would be unstable in the presence of external perturbations. As a future study, it might be interesting to calculate the decay rate of such quantum solitons by introducing small perturbations to the DNLS Hamiltonian.

Recently, we have discovered additional ranges of values of $\phi$ for which there are bound and anti-bound $N$-soliton states. The complete description of these ranges can be found using concepts from number theory such as Farey sequences and continued fractions [20].

\section{References}

1. H. B. Thacker, Rev. Mod. Phys. 53 (1981) 253.

2. L. D. Faddeev, Sov. Sci. Rev. C 1 (1980) 107; in Recent Advances in Field Theory and Statistical Mechanics, ed. J.-B. Zuber and R.Stora, (North-Holland, Amsterdam, 1984 ) p.561.

3. E. K. Skylanin, in Yang-Baxter Equation in Integrable systems, Advanced series in Math. Phys. Vol. 10, edited by M. Jimbo (World Scientific, Singapore, 1990) p.121.

4. E. K. Skylanin, L. A. Takhtajan and L. D. Faddeev, Theor. Math. Phys. 40 (1980) 688.

5. G. Bhattacharya and S. Ghosh, Int. J. Mod. Phys. A 4 (1989) 627.

6. A. G. Shnirman, B. A. Malomed and E. Ben-Jacob, Phys. Rev. A 50 (1994) 3453.

7. D. Sen, Quantization of the derivative nonlinear Schrödinger equation, cond-mat/9612077.

8. A. Kundu and B. Basu-Mallick, J. Math. Phys. 34 (1993) 1052.

9. B. Basu-Mallick and T. Bhattacharyya, Nucl. Phys. B 634 [FS] (2002) 611.

10. B. Basu-Mallick and T. Bhattacharyya, Jost solutions and quantum conserved quantities of an integrable derivative nonlinear Schrödinger model, hep-th/0304063. 
11. M. Wadati, H. Sanuki, K. Konno and Y.-H. Ichikawa, Rocky Mountain J. Math. 8 (1978) 323; Y.-H. Ichikawa and S. Watanabe, J. de Physique 38 (1977) C 6-15.

12. P. A. Clarkson, Nonlinearity 5 (1992) 453.

13. M. Rosenbluh and R. M. Shelby, Phys. Rev. Lett. 66 (1991) 153.

14. U. Aglietti, L. Griguolo, R. Jackiw, S.-Y. Pi and D. Seminara, Phys. Rev. Lett. 77 (1996) 4406; R. Jackiw, A nonrelativistic chiral soliton in one dimension, hep-th/9611185.

15. S. J. Benetton Rabello, Phys. Rev. Lett. 76 (1996) 4007; (E) 77 (1996) 4851.

16. H.H. Chen, Y.C. Lee and C.S. Liu, Phys. Scr. 20 (1979) 490.

17. D.J. Kaup and A.C. Newell, J. Math. Phys. 19 (1978) 798.

18. H. Min and Q.-H. Park, Phys. Lett. B 388 (1996) 621.

19. E. Gutkin, Ann. Phys. 176 (1987) 22.

20. B. Basu-Mallick, T. Bhattacharyya and D. Sen, under preparation. 